\title{
SEGMENTATION AND RECONSTRUCTION OF BUILDINGS WITH AERIAL OBLIQUE PHOTOGRAPHY POINT CLOUDS
}

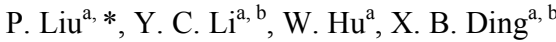 \\ ${ }^{a}$ China TopRS technology Co.Ltd., Beijing, China - liupei@casm.ac.cn \\ ${ }^{\mathrm{b}}$ Chinese Academy of Survey \& Mapping, Beijing, China - casmrsec@sina.com
}

Commission VI, WG VI/4

KEY WORDS: Oblique photography, stereo matching, point clouds segmentation, building reconstruction

\begin{abstract}
:
Oblique photography technology as an excellent method for 3-D city model construction has brought itself to large-scale recognition and undeniable high social status. Tilt and vertical images with the high overlaps and different visual angles can produce a large number of dense matching point clouds data with spectral information. This paper presents a method of buildings reconstruction with stereo matching dense point clouds from aerial oblique images, which includes segmentation of buildings and reconstruction of building roofs. We summarize the characteristics of stereo matching point clouds from aerial oblique images and outline the problems with existing methods. Then we present the method for segmentation of building roofs, which based on colors and geometrical derivatives such as normal and curvature. Finally, a building reconstruction approach is developed based on the geometrical relationship. The experiment and analysis show that the methods are effective on building reconstruction with stereo matching point clouds from aerial oblique images.
\end{abstract}

\section{INTRODUCTION}

It comes as no surprise to find out that cities are the main places of human activity, and also the focus and core of regional socioeconomic development. As geographic information of the buildings has more and more relevance to human beings, it is important for the research on reconstruction of the building geometry. In recent years, the development of the oblique photography technology makes possible rapid construction of three dimension models of cities. Tilt and vertical images with high overlaps and different visual angles can produce a large number of dense matching point clouds data with spectral information. There are some methods of segmentation and reconstruction buildings with dense point clouds. Most of them based on geometrical characteristics such as curvature and normal often lead to over-segmentation or even failure. Several methods based on colors are hard to achieve satisfactory global segmentation effects, because the three color components are relative and influenced by lighting and shading factors in the scene. Based on these observations, this paper combines the geometrical characteristics and colors information to segment and reconstruct buildings.

\section{SEGMENTATION}

Segmentation is fundamental work for building reconstruction, which refers to the grouping points with similar properties. Buildings can be modelled by a data driven approach based on generic assumptions such as planarity of the surface. Several algorithms and their characteristics for segmentation of point clouds are discussed below:

In clustering algorithms, the parameter is first carried out for each roof point of a building, and then the points will be clustered with homogenous surfaces, such as planes, with computer vision pattern recognition algorithm for segmentation. But this algorithm needs the prior knowledge of building which is always difficult to obtain, so the cluster centre is unstable, and the algorithm is too complicated .

Hough transform algorithm is primarily used in the twodimensional data processing and later extended to the threedimensional data processing, which transforms the plane from Euclid space to the parameter space. It generates all possible planes according to the point clouds, later the segment of planes that reach certain number of points are regarded as the segmentations. However, this process results in sawtooth planes when processing point clouds, and the processing time and the memory appropriated will be high.

RANSAC algorithm is applied to segmentation of point clouds, which consists to search the best plane among $3 \mathrm{D}$ point clouds. It fist randomly and iteratively samples three points necessary to determine the plane parameters. Then it detects the rest of the point clouds belonging to the calculated plane, according to a given threshold. Afterwards, it repeats these procedures $\mathrm{N}$ times; and obtains the best plane among $\mathrm{N}$ planes according to defined criteria. The processing time of this algorithm is low, but the best plane that it detected may be not always represent a roof plane, because it doesn't consider the local connectivity of point clouds and the geometry of a roof.

In region growing algorithm, seed points must be selected for point clouds of segmentation region, and then we use the similarity between a given set of point clouds to group the points. Effective segmentation can be obtained, but it is difficult to select the seed points and set parameters, and the region growing process is easy affected by noise points, such as roof dormer, chimneys.

* Corresponding author 
The stereo matching point clouds from aerial oblique images may have several defects, such as uneven distribution, point hollows and low precision which will cause points fluctuation in a plane. Therefore, the segmentation methods for stereo matching point clouds should have some faults. Firstly, segmentation methods will have a larger number of parameters which it is difficult to set. Secondly, there are some modelbased approaches to segmentation such as RANSAC, 3D Hough Transform algorithm will produce fake planes and marginal noise points. Thirdly, some segmentation algorithms are only based on geometrical derivatives or colors which are not full use of the information of stereo matching point clouds. In this paper, we presented is based on curvature, normal and colors. Normal estimation, colorimetrical difference measurement and region growing algorithm details are explained below:

\subsection{Normal estimation}

The normal for each point is estimated by fitting a plane to some neighboring points. So the normal of point is affected by neighborhood selection, we selected neighborhood in $\mathrm{K}$ nearest neighborhood (KNN)or fixed distance neighbors (FDN) methods, which can be optimized using k-d trees to organize point clouds. When the point clouds are uneven distribution, we often search for KNN to obtain neighbors.

After neighborhood selection, suppose the KNN search of neighbors $\mathrm{Kp}$ consists of a set of $\mathrm{mp}$ points $\left\{X_{i}\left(i=1, \ldots, m_{p}\right)\right\}$.Let $\bar{X}$ be their mean vector, then the covariance matrix $\mathrm{C}$ is calculated as

$$
C=\sum_{i=1}^{m_{p}}\left(X_{i}-\bar{X}\right)\left(X_{i}-\bar{X}\right)^{T}
$$

For this covariance matrix $C$, its eigenvalues $\lambda 1, \lambda 2, \lambda 3(\lambda 1 \leqslant \lambda 2$ $\leqslant \lambda 3)$ and the curvature $\lambda$ can be determined as

$$
\lambda=\lambda_{1} /\left(\lambda_{1}+\lambda_{2}+\lambda_{2}\right)
$$

\subsection{Colorimetrical difference measurement}

We measure colorimetrical difference in RGB space by Euclidean distance. The colorimetrical difference function is defined as:

$$
d\left(c_{1}, c_{2}\right)=\sqrt{\left(r_{1}-r_{2}\right)^{2}+\left(g_{1}-g_{2}\right)^{2}+\left(b_{1}-t\right.}
$$

Where $\mathrm{c} 1$ and $\mathrm{c} 2$ represent color vectors of two points.

\subsection{Region growing}

\begin{tabular}{l}
\hline $\begin{array}{l}\text { Algorithm segment a given point cloud using normal } \\
\text { curvature and colors information. }\end{array}$ \\
\hline Inputs: Point clouds $=\{\mathrm{P}\}$, point normals $\{\mathrm{N}\}$, point colors \\
$\{\mathrm{C}\}$, curvature $\{\mathrm{r}\}$, neighbor finding function $\Omega()$, \\
curvature threshold $\mathrm{r}_{\mathrm{th}}$, angle threshold $\theta_{\mathrm{th}}$, colorimetrical \\
difference calculation function $\mathrm{d}\left(\mathrm{c}_{1}, \mathrm{c}_{2}\right)$, point \\
colorimetrical similarity threshold $\mathrm{T}_{\mathrm{pp}}$.
\end{tabular}

In the region growing processing, we firstly specify a curvature threshold rth, angle threshold th, point colorimetrical similarity threshold Tpp. The point with minimum curvature is selected as the current seed. Then, the neighboring points of the current seed are determined with KNN or FDN, we using KNN instead of FDN because the point clouds are uneven distribution. The points that angle threshold is less than th or colorimetrical difference is less than Tpp, add them to current region. The points whose curvatures are less than rth add them to the list of potential seed points. If the potential seed point list is not empty, set the current seed to the next available seed. Add the current region to the segmentation, until all the point clouds are segmented.

\section{BUILDING RECONSTRCUTION}

Building reconstruction is carried out after segmentation of building roofs. For a building reconstruction, segment boundary points are first traced by a local convex hull formation approach. In the second step, boundary points are determined which points are in the same segment edge. In the third step a least squares model is used to regularize segment edges to form shape of the segment. Finally, we restore the relationship among every segment shape to reconstruct building. To form the shape of segment is described as follows:

\subsection{Boundary Tracing}

A local convex hull algorithm is applied to obtain boundary points. For a given set of points of segment, the left-most point which has minimum $\mathrm{x}$ is the convex hull point of the segmentation. Then all points that lie within a threshold distance are selected, the threshold distance is according to the point density of the point clouds. The point that is the first convex hull point in local points is regarded as the convex hull 
point. Repeat it until the convex hull point is the same as the left-most point, the boundary points are traced.

\subsection{Boundary Grouping}

Boundary points that are located on the same boundary are divided into a group. The main step is to find the key points of the boundary points. If the angle between the point with its preadjacent point and its post-adjacent point is beyond a certain angle, the point is the key points. Therefore the points between two key points are in the same boundary, grouped as a group.

\subsection{Boundary Regularization}

A least squares model is used to fit the points in every group. (Assuming that most buildings have only two dominant directions that are also mutually perpendicular, we can use the points to determine these directions, and fit parametric lines that represent the edge or the footprint of the building.) The dominant direction of boundary should be determined first. Then we can adjust the every fitted boundary according to this rule:(1)if the boundary whose direction is similarly parallel to dominant direction should be adjusted its direction same with the dominant direction.(2)if the boundary whose direction is similarly perpendicular to dominant direction should be adjusted its direction same with the perpendicular direction.(3)the rest of boundary needn't be adjusted. Finally the shape of segment is formed.

After getting the shape of segment, the relationship among the shapes should be restored to reconstruct the buildings. As the segmentation is not accurate enough and boundary regularization exits errors, The public edges may not coincide with each other, which intersect with a small angle or are parallel with a certain distance. Therefore, the public edges must be adjusted. when the edges are same in R2, they are the building roof lines that are the same edge if the elevation difference is small in a certain threshold distance, else they are the building step lines. The roof lines should be adjusted based on averages of the two roof lines. The step lines should not be adjusted.

\section{RESULTS}

The presented solution framework for the segmentation and reconstruction was applied to point clouds. The point clouds are produced with Street Factory software with aerial oblique images obtained from TopRS-DC5 developed by ChinaTopRS Company in china as input data. The image of test area is displayed in Figure1(a), and the point clouds are showed by elevation in Figure1(b), by point color in Figure1(c). The segmentation results are represented with the algorithm we presented from above in Figure1(d), The different color points are represented different segments excepted that the segments with less points are labeled white. For those results the th was set to $18^{\circ}$ and the Tpp was set to 30 , using KNN search neighbors with $\mathrm{k}=8$. rth was automatically calculated by the 95th percentile of the curvature. In the results we see the segmentation results are good oval, although there are areas where are not segmented over-segmentation occurred, e.g., the red rectangle in Figure1(d). The effect of segmentation is related to the parameters th, Tpp and rth. Result of buildings reconstruction is showed in Figure1(e), we can see the result of reconstruction is affected by the effects of segmentation. If the segmentation is lack of points, some parts of buildings marked

by yellow circles are missing. The arc roofs cannot be reconstructed, flat roofs are replaced, e.g., the green rectangles. The buildings with inner annulus are not reconstructed correctly, showed in blue rectangles.

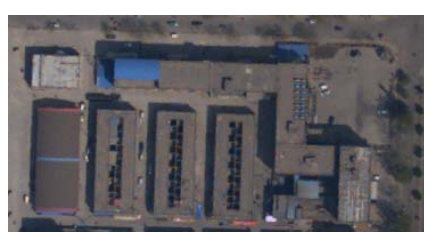

(a)

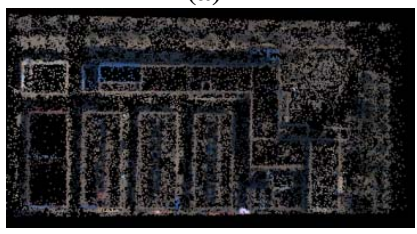

(c)

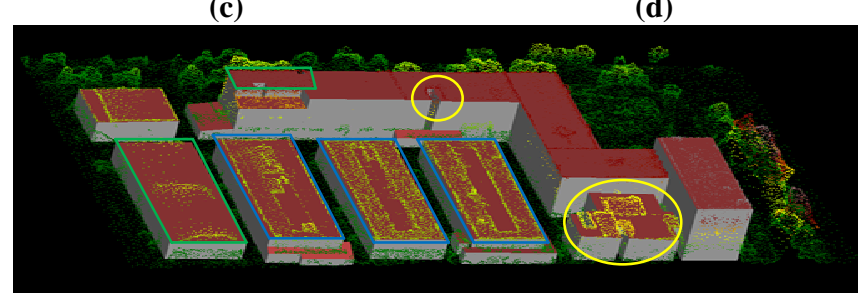

(e)

Figure1. Result of segmentation and reconstruction (a)image of test area (b) point clouds by elevation (c)point clouds by color

(d)result of segmentation (e)result of reconstruction

\section{CONCLUSIONS}

A solution framework for the segmentation and reconstruction of building roofs using stereo matching point clouds from oblique images has been represented. The segmentation algorithm is based on geometrical derivatives and colorimetrical similarity, which use surface normal, curvature and RGB colors information. The curvature is used to judge seed points of segmentation, the point that normal and RGB colors satisfy condition will be added to the segments. Therefore, this method can eliminate the influence of point clouds fluctuations and noise points and can achieve satisfying segmentation. However, effects of segmentation depend on the value for th, Tpp, rth. The building reconstruction is achieved with forming the shape of every segment and restoring the relationship of the segments. The presented method can't reconstruct the building with arc roofs, or inner annulus. And the correctness of building reconstruction is affected by the effects of segmentation. The results of building reconstruction are showed that the solution framework is effectiveness and feasible.

\section{ACKNOWLEDGEMENTS (OPTIONAL)}

We gratefully acknowledge the financial support of Special Fund for Surveying, Mapping and Geoinformation Research in the Public Interest(201412019)and National HighTechnology Research and Development Program("863"Program) of China(2013AA122104). 


\section{REFERENCES}

G. Vosselman and S. Dijkman, "3D building model reconstruction from point clouds and ground plans," Int. Arch. Photogramm. Remote Sens.,vol. 34, no. 3/W4, pp. 37-43, 2001.

Filin S, Pfeifer N. Segmentation of airborne laser scanning data using a slope adaptive neighborhood.ISPRS Journal of Photogrammetry and Remote Sensing, 2006, 60(2): 71-80.

Rabbani, T., van den Heuvel, F.A. and Vosselman, G., 2006. Segmentation of point clouds using smoothness constraint.In: International Archives of the Photogrammetry, Remote Sensing and Spatial Informantion Sciences, Vol. 36, Dresden, Germany,pp.248-253.

Forlani, G., Nardinocchi, C., Scaioni, M. and Zingaretti, P.,2004. Building reconstruction and visualization from Lidar data.Int. Arch. of the Photogrammetry, Remote Sensing and Spatial Information Sciences, Vol. XXXIV, Part 5/W12.

Q.M.Zhan., Y.B.Liang and Y.H.Xiao, "Color-based segmentation of point clouds".In: Bretar F, Pierrot-Deseilligny M, Vosselman G (Eds) Laser scanning 2009, IAPRS, Vol. XXXVIII, Part 3/W8 - Paris, France, September 1-2, 2009.

A. Sampath and J. Shan, "Segmentation and reconstruction of polyhedral building roofs from aerial Lidar point clouds". IEEE Trans. Geoscience and Remote Sensing, vol. 48, no. 3,pp.15541567,Mar. 2010.

F. Tarsha-Kurdi, T. Landes, and P. Grussenmeyer, "Houghtransform and extended RANSAC algorithms for automatic detection of 3D building roof planes from LiDAR data," in Proc. Int. Soc. Photogramm. Remote Sens., 2007, vol. 36, pp. 407412.

Ameri, B. and Fritsch, D., 2000. Automatic 3D building reconstruction using plane-roof structures, Published by the ASPRS, Washington DC.

V. Verma, R. Kumar, and S. Hsu, "3D building detection and modeling from aerial LiDAR data," in Proc. IEEE Comput. Soc. Conf. CVPR, 2006,vol. 2, pp. 2213-2220.

A.Bab-Hadiashar and N. Gheissari, "Range image segmentation using surface selection criterion," IEEE Trans. Image Process., vol. 15, no. 7,pp. 2006-2018, Jul. 2006.

A. Sampath and J. Shan, "Building boundary tracing and regularization from airborne LiDAR point clouds," Photogramm. Eng. Remote Sens., vol. 73, no. 7, pp. 805-812, 2007. 